\title{
A polynomial equation to predict low back compression force: accounting for the effects of load height on instability
}

\author{
Inger Christina Calder ${ }^{\mathrm{a}}{ }^{*}$, Jim R. Potvin \\ ${ }^{a}$ Mayo Clinic, 200 First Street SW, Rochester, Minnesota, USA, 55905 \\ ${ }^{\mathrm{b}}$ Department of Kinesiology, McMaster University, Hamilton, Ontario, Canada
}

\begin{abstract}
The purpose of this study was to develop a regression equation that, incorporating the potential energy of the load in the hands, was capable of improved predictions of spinal compression forces. A stepwise polynomial equation was developed from EMG profiles of 15 muscles, and its spinal joint loading predictions at L4/L5 were compared to current methods of calculating spinal compression. Absolute muscle activation was shown to increase with increased loading height, indicating that the central nervous system responds to changes in spinal stability. The inclusion of potential energy into the calculation of spinal disc compression at L4/L5 improved estimates of the compressive forces acting on the spine. This is the first model to incorporate potential energy into a predictive model for lumbar spine compression without the use of electromyography. It was concluded that potential energy plays a vital role in dictating the recruitment patterns of the trunk.
\end{abstract}

Keywords: lifting, compression, low back loading

* Corresponding author. E-mail: calder.inger@mayo.edu Tel: +1 5072662774 


\section{Introduction}

In an attempt to further understand the etiology of back pain, several researchers have developed biomechanical models to predict spinal compression force. These models can be used to assess manual material handling tasks, and the results are used to guide task design and reduce the risk of low back injury [2]. Methods that are currently available for calculating compression force include: single equivalent models, EMG-based models, optimization methods [6] and polynomial equations [10]. Each previous method has based estimates of muscle force solely on reaction moments, with no consideration of destabilizing potential energy of the $\operatorname{load}(\mathrm{s})$. Previously, two other models used stability as a criterion in their optimization scheme to improve compression force estimates, but they are not suitable for field use [1,12]. Those models indicated that a consideration is needed of the delicate interplay between compression and stability. Unfortunately, these models required the use of EMG and were not practical for field use.

The aim of the present study was, thus, to determine if it is feasible that a regression equation, based on experimental data and including load height, would be a useful ergonomic tool that is easy to use and widely applicable. We present work showing how to incorporate stability in a simple practical equation that uses easily measured field variables to estimate L4/L5 compression force, during asymmetrical, isometric loading tasks.

\section{Method}

\subsection{Study design}

The study aimed to develop a polynomial equation that uses moment and potential energy to predict joint compression forces at the L4/L5 level. The compression output from three existing biomechanical models was also calculated for comparison during 48 loading tasks (36 vertical, \& 12 triaxial loading tasks). The selected biomechanical models of the spine provided an estimation of joint compression and shear forces, by distributing the moment generated about L4/L5 amongst the musculature of the trunk. Electromyographic (EMG) data were collected from 15 muscles during static load handling tasks. In addition, various types of biomechanical models of the spine were utilized to develop a simple equation that has more biological fidelity than previous statistical models used to predict lumbar joint loading during asymmetrical tasks.

\subsection{Participants}

Sixteen healthy right-handed ( 8 female and 8 male, age $24.3 \pm 1.5$ years, body mass $76.6 \pm 12.6 \mathrm{~kg}$ and stature $178.1 \pm 8.36 \mathrm{~cm}$ ) participated in a repeated measures experimental design approved by the Research Ethics Board of the University of Windsor.

\subsection{Experimental protocol}

Participants performed various one-handed static loading tasks, in a laboratory setting using their right hand. A total of 36 three-dimensional vertical loading conditions, and 12 triaxial loading conditions were examined.

For the vertical loading trials, subjects held a load in 18 different locations for each of 2 vertical loading moment conditions. $(6.7$ or $13.3 \mathrm{Nm})(n=36)$. Using the acromion as a reference point, a framework of eighteen targets was suspended from an overhead apparatus. Participants were asked to stand with their hips pressed to a foam-padded saddle. All hand locations were standardized to an absolute vertical distance from the L4/L5, as well as an absolute distance from the right acromion. Platforms were used as required.

The targets were placed at 3 heights $(10,40$, and $70 \mathrm{~cm}$ above L4/L5), 3 angles of transverse shoulder rotation $\left(0^{\circ}, 45^{\circ}\right.$ and $90^{\circ}$ from the midline), and 2 distances from the acromion $(0.25$ and $0.50 \mathrm{~m})$ and 2 different moment conditions.

Additionally, for the sake of formulating regression equations, exertions causing moments about all three axes of the spine were performed during an additional 2 sets of triaxial loading trials. This subset of exertions was performed at 3 heights, 1 shoulder angle $\left(0^{\circ}\right)$, and 2 reach distances, for a total of 2 different triaxial moments $(n=12)$.

The triaxial hand loading trials were achieved by utilizing 2 different pulley systems. The first set of triaxial loading trials, Triaxial-1, required the subject to perform a hand effort down, forward and to the right. The second set, Triaxial-2, required the subject to perform a hand effort up, backward and to the left.. The resisted load was $44.5 \mathrm{~N}$ for both triaxial conditions. 
All 48 testing conditions were presented in a randomized order. Each trial was held for 3 seconds, and repeated three times. Two minutes rest was provided between trials and data collection took place during one session.

\subsection{Data acquisition}

Surface EMG (sEMG) was collected from 15 muscles using bipolar disposable $\mathrm{Ag}-\mathrm{AgCl}$ surface electrodes with an inter-electrode distance of $2.5 \mathrm{~cm}$. The muscles examined were the bilateral lumbar erector spinae (LES), thoracic erector spinae (TES), multifidus (MULT), latissimus dorsi (LD), rectus abdominus (RA), internal oblique (IO), and external oblique (EO), and also from the right anterior deltoid (RDELT).

A quiet EMG trial was collected and used to remove signal bias from EMG signals prior to processing. A series of muscle-specific maximum voluntary contraction trials were performed and used to normalize EMG. Maximum voluntary excitation (MVE) was determined as average activity over a $500 \mathrm{~ms}$ window about the peak activation for each muscle.

\subsection{Data analysis}

sEMG data were collected with LabView software (National Instruments, Austin, TX) using a PC compatible computer and converted by a 12-bit A/D card (National Instruments, Austin TX). All sEMG signals were processed through a differential amplifier (gain $=1000$ to 5000 , input impedance $=10$ $\mathrm{G} \Omega \mathrm{s}, 10-1000 \mathrm{~Hz}, \mathrm{CMRR}=115 \mathrm{~dB}$ at $60 \mathrm{~Hz}$, Bortec, Octopus AMT-8, Calgary, Canada). sEMG signals were digitally sampled at $2048 \mathrm{~Hz}$. EMG data were then bandpass filtered $(20-1000 \mathrm{~Hz})$, full wave rectified, and then low-pass filtered using a $2^{\text {nd }}$ order Butterworth filter with a frequency cutoff of $2 \mathrm{~Hz}$.

\subsection{Statistics}

A 3-way Repeated Measures ANOVA was performed to determine the main effects and interactions of the independent variables on EMG amplitude. The independent variables were: 1) load height, 2) shoulder angle, and 3) moment condition. The dependent variable was the normalized EMG amplitudes for each monitored muscle. Tukey's Posthoc analyses were run to interpret any significant main effects or interactions that were found.

\subsection{Development of the equation}

A forward stepwise regression was performed using the height of the subject, the height of the load above the L4/L5 level, body mass, the flexion moment acting at the L4/L5 level, the lateral moment acting at the L4/L5 level, the axial moment acting at the L4/L5 level, as well as squared and cubed values of each of the aforementioned moments.

The three moments at L4/L5, and the load potential energy from the time-histories of all subject-trials, were input as variables in StatView (SAS Institute Inc., 1992-1998) software. Using the experimentally derived EMG profile of each muscle, the EMG amplitude was averaged for each loading condition, for all subjects, across all trials $(n=48)$ and utilized as input for the reference model, the EMGoptimization model of Cholewicki et al, [6]. The output was selected as the criterion, as it was based on EMG recorded from the trunk muscles and was sensitive to between and within subject differences for each trial of each condition.

\subsection{Estimation of compression values}

Lumbar compression force was calculated for each of the 48 conditions with 3 biomechanical approaches: 1) Cholewicki and McGill EMG-assisted Optimization hybrid model (EMGAO) [3], 2) McGill, Norman and Cholewicki 3rd order polynomial equation [4], and 3) Michigan 3Dimensional Static Strength Prediction Program (3DSSPP).

3DSSPP software was used to determine the reaction moments and forces about L4/L5 by inputting external hand forces and force vectors for desired anthropometry. This was achieved by manipulating the Michigan 3-D mannequin into the body postures adopted by the subjects in order to replicate joint kinematics. The reaction moments were then used as input to other models, to calculate the compressive forces to be compared between spine models.

The compression values generated by the new polynomial were compared against those predicted by the common compression estimation techniques to evaluate the performance of this model. All comparisons included correlations, and RMS differences between the biomechanical methods. 


\section{Results}

\subsection{Effect of load height}

Increased load height resulted in a generalized increase in myoelectric activity in 14 of the 15 monitored muscles, with the exception of the left multifidus, when the average EMG amplitude was pooled across angle and moment conditions. Relative to the condition where the load was held $10 \mathrm{~cm}$ above the L4/L5 level (low-height), the muscles on the right side of the body increased their activity approximately $148 \%$ at $40 \mathrm{~cm}$ (mid-height), and $212 \%$ at $70 \mathrm{~cm}$ (high-height). The height effect was also significant for the muscles on the left side of the body; however, the increase in EMG amplitude was not as high. When pooled across angle and moment conditions the muscles on the left side of the body demonstrated increases of approximately $114 \%$ at 40 $\mathrm{cm}$, and $142 \%$ at $70 \mathrm{~cm}$ above the L4/L5 joint.

\subsection{Regression equation}

The forward stepwise regression identified the following variables as being critical in the prediction of spinal joint loading under the current experimental settings: the height of the subject, the flexion moment acting on the subject at L4/L5, the lateral moment acting on the subject at L4/L5, and the axial moment acting on the subject at L4/L5, as well as squared and cubed values of each variable. Eq. (1) shows the regression equation, resulting from the stepwise regression to predict L4/L5 compression.

Where:

- COMP $=$ Compression $(\mathrm{N})$

- LH = external load height above L4/L5 (cm)

$-\mathrm{SH}=$ Height of the subject $(\mathrm{cm})$

$-\mathrm{M}_{\mathrm{F}}=\mathrm{L} 4 / \mathrm{L} 5$ flexion moment $(\mathrm{Nm})$

- $\mathrm{M}_{\mathrm{L}}=\mathrm{L} 4 / \mathrm{L} 5$ lateral bend moment $(\mathrm{Nm})$

$-\mathrm{M}_{\mathrm{A}}=\mathrm{L} 4 / \mathrm{L} 5$ axial twist moment $(\mathrm{Nm})$

\subsection{Regression model performance}

The regression-based polynomial model of the present study predicted compression values with the closest agreement to the experimental data (RMS error $=291 \mathrm{~N}, \mathrm{r}^{2}=0.43, \mathrm{n}=48$ ), followed by the polynomial equation of McGill et al. (1996) (RMS error $\left.=321 \mathrm{~N}, \mathrm{r}^{2}=0.29\right)$ and the posture prediction software 3DSSPP (RMS error of $600 \mathrm{~N}, \mathrm{r}^{2}=0.20$ ) The RMS errors were found to be the lowest at Low heights and highest at the High heights for the prediction of compressive forces for all methods (Figure 1).

\section{Discussion}

Previous ergonomic methods, utilized for predicting spinal compression force, have used estimations of muscle forces that are based solely on moments. Research has shown that controlling the moment arm, the load magnitude or the anatomical model will have an effect on the predicted compression [5,7]. Theoretically, these models would not show an increase in predicted muscle forces if the effective moment arm remained constant, and the load height increased.

Research has shown that the motor control system responds to stability requirements in addition to moment equilibrium requirements [4,8]. Two existing optimization models use stability as a criterion when predicting compression [11,12], however, these models do not isolate load height as a variable that may affect the prediction of compressive forces in the lumbar spine.

Despite the limitations of this study, the results agree with previous research which states that the CNS responds to changes in the biomechanical spinal stability of the trunk [9]. By independently influencing the potential energy of the system and trunk moment, this study shows that the inclusion of load height may improve predictions of spinal load predictions.

The experimental trials were designed so that the

$$
C O M P=3326.64+3.81(L H)-16.68(S H)+11.81\left(M_{F}\right)+0.68\left(M_{F}^{2}\right)+0.56\left(M_{L}\right)^{2}-0.06\left(M_{A}{ }^{3}\right)
$$




\section{EMG Based Compression vs. Predicted Compression from 3 Modeling Methods}

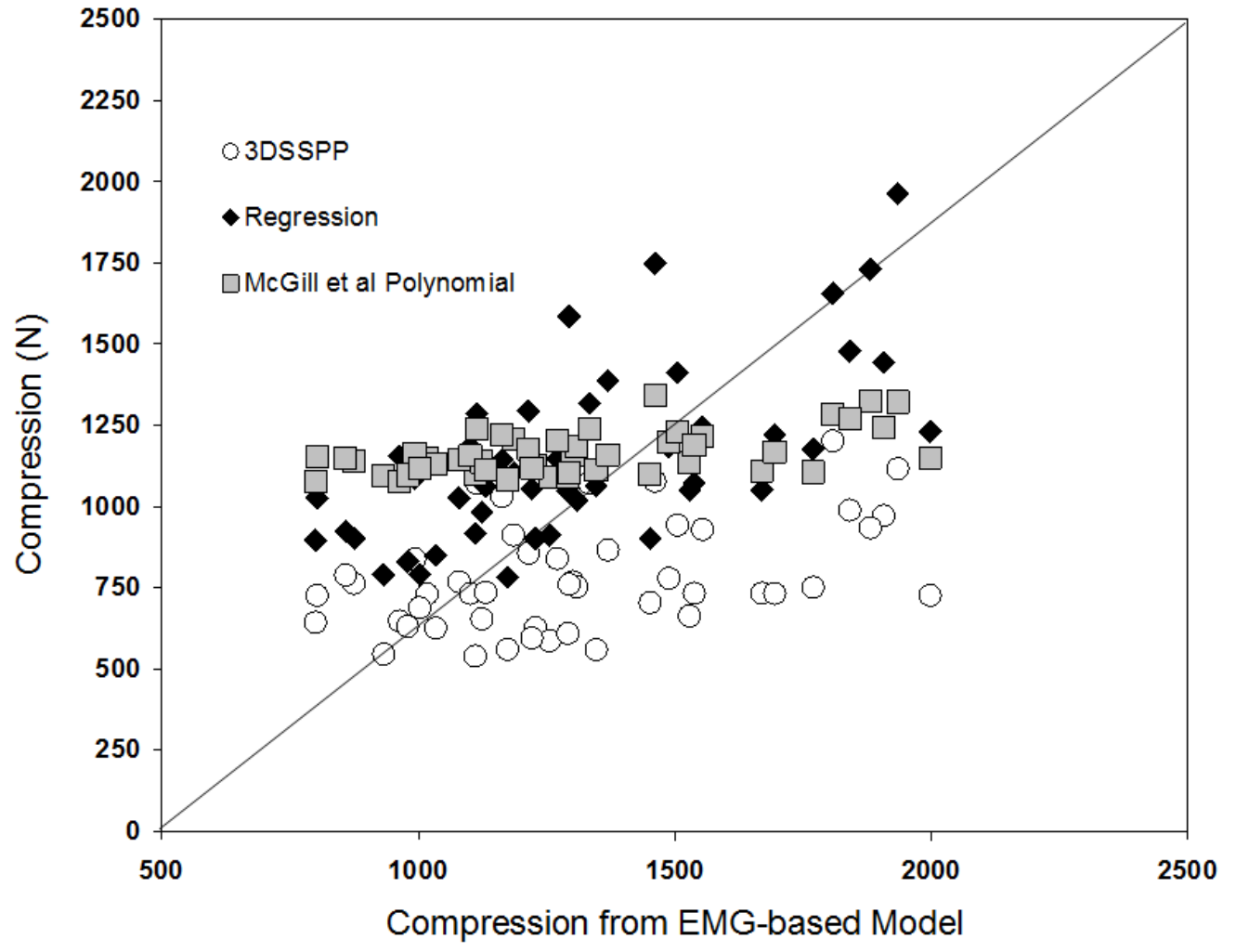

Fig. 1 Comparisons were made between the compression values calculated by each of the 4 models; 3DSSPP, the polynomial of McGill et al. (1996), the regression equation developed in this study, and lastly, the EMG-optimization model [3], which is displayed on the $\mathrm{x}$ axis. $\mathrm{N}=48$ for all.

external moment would be similar for two conditions, at two separate moment arms. This allowed the potential energy of the system to be altered while the load moment was controlled. In this manner, the external moment acting on the subject did not change substantially. Thus, prospective muscle activation pattern changes would implicate a modified stability scenario to which the motor control system was responding.

The current regression-based model was found to correlate very well with the measured myoelectric activity. This relationship was established by comparing the output from the various predictive methods with the compression predicted by the EMGAO method [3]. Comparisons were made between the compression values calculated by each of the four methods; 1) 3DSSPP, 2) the polynomial equation of McGill et al. [10], 3) the regression equation developed in this study, and 4) the EMGoptimization model [3].
With the current model, average RMS errors were shown to be lower than those of other models used to predict spinal compression forces in industry. This resulted because of the novel inclusion of loading height as a variable in the calculation. The significant increases in antagonist and antagonistic trunk muscle activity observed when load height was increased and the external moment remained relatively unchanged. This indicates that the CNS is responsive to factors other than moment arm and load magnitude when generating the optimal activation pattern in order to satisfy the 6 equations of equilibrium in isometric conditions. Therefore, incorporating loading height into the predictive measures of spinal loading promotes a more realistic representation of actual spine compression.

During the more complex loading scenarios, such as the triaxial moment conditions of this study, the role of the muscles acting to stabilize the spine becomes increasingly obscure. It is possible that the 
role of any given muscle is twofold; generate a restorative moment (agonist), and counteract the moments generated by the agonists in order to stabilize the joint that is threatened by instability by the agonists that would further perturb the spine in the direction of the original perturbation (antagonist).

\section{Conclusion}

The current study has demonstrated in principle that, when predicting spinal compression, the inclusion of the external hand load height (or potential energy) as an input variable significantly improves the prediction of spinal compressive forces.

Further work is necessary in the field of spinal stability; specifically, in the modeling of stability of the spinal system. Models should be developed to encompass the entire spine as a system and include the effects of intra-abdominal pressure, the thoracolumbar fascia, and the transversus abdominis. This will provide a more realistic representation of compression in the human lumbar spine. These models should ultimately be validated with in vivo measurements of compression, rather than comparing the output to that of similar models.

Additional research is needed to test trunk muscles under systematically varied levels of stability, in which external moments are applied to more than one axis simultaneously. In the current study, this was done but with only 12 of 48 sets of loading conditions. The current model should be tested under more complex loading conditions using load magnitudes that are more characteristic of those seen in the industrial setting, and also loading scenarios that are dynamic in nature. Although it was intended that the loads incorporated into the current experimental design did not produce compression loads in the range of the NIOSH limit [13], a great deal of insight into neuromotor recruitment of muscle activity, spinal stability and lifting biomechanics could be gained through the investigation of this model with a range of heavier loads.

While this equation is not presently being suggested for use in the field, the authors believe this work provides a proof in principle that future efforts to estimate compression in field models (where it is not feasible to measure EMG) should include height of the load in addition to the moments. The future studies will likely have to have higher loads and be dynamic in nature. Further investigation is warranted to explore the feasibility of incorporating the effect of the potential energy of a load into existing ergonomic analyses software programs used for calculating joint compression forces. This could potentially be achieved with a similar regressionbased equation that would be used as a supplemental tool to the software.

All of the aforementioned advances in spinal modeling would unquestionably provide invaluable insight into the nature of spine instability and buckling, and discern those loading parameters that are most likely to cause tissue damage in the low back.

\section{References}

[1] Brown, S. H. \& Potvin, J. R. (2007). Exploring the geometric and mechanical characteristics of the spine musculature to provide rotational stiffness to two spine joints in the neutral posture. Hum.Mov Sci., 26, 113-123.

[2] Chaffin, D. B. (2005). Primary prevention of low back pain through the application of

[3] Cholewicki, J. \& McGill, S. M. (1994). EMG assisted optimization: a hybrid approach for estimating muscle forces in an indeterminate biomechanical model. J.Biomech., 27, 1287-1289.

[4] Cholewicki, J. \& McGill, S. M. (1996). Mechanical stability of the in vivo lumbar spine: implications for injury and chronic low back pain. Clin.Biomech., 11, 1-15.

[5] Cholewicki, J. \& VanVliet, J. J. (2002). Relative contribution of trunk muscles to the stability of the lumbar spine during isometric exertions. Clin.Biomech., 17, 99-105.

[6] Cholewicki, J., McGill, S. M., \& Norman, R. W. (1995). Comparison of muscle forces and joint load from an optimization and EMG assisted lumbar spine model: towards development of a hybrid approach. J.Biomech, 28, 321-331.

[7] Cholewicki, J., Simons, A. P., \& Radebold, A. (2000). Effects of external trunk loads on lumbar spine stability. J.Biomech., 33, 1377-1385.

[8] Gardner-Morse, M., Stokes, I. A., \& Laible, J. P. (1995). Role of muscles in lumbar spine stability in maximum extension efforts. J.Orthop.Res., 13, 802-808.

[9] Granata, K. P. \& Orishimo, K. F. (2001). Response of trunk muscle coactivation to changes in spinal stability. J.Biomech., 34, 1117-1123.

[10] McGill, S. M., Norman, R. W., \& Cholewicki, J. (1996). A simple polynomial that predicts low-back compression during complex 3-D tasks. Ergonomics, 39, 1107-1118.

[11] Potvin, J. R. \& Brown, S. H. (2005). An equation to calculate individual muscle contributions to joint stability. J.Biomech., 38, 973-980

[12] Stokes, I. A. \& Gardner-Morse, M. (2001). Lumbar spinal muscle activation synergies predicted by multi-criteria cost function. J.Biomech., 34, 733-740.. Newman and E.T. Liu, Perspective on BRCA1, Breast Disease 10 (1998), 3-10.

[13] Waters, T. R., Putz-Anderson, V., Garg, A., \& Fine, L. J. (1993). Revised NIOSH equation for the design and evaluation of manual lifting tasks. Ergonomics, 36, 749-776. 